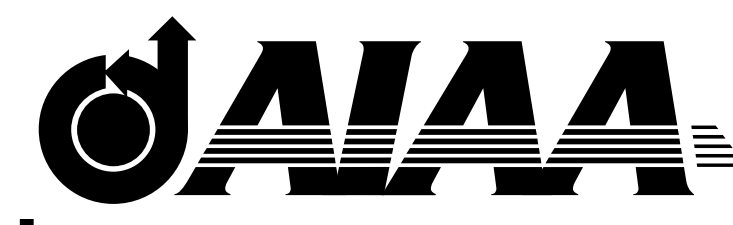

\title{
AIAA 2003-1747
}

\section{Nonlinear Vibration of Wrinkled Membranes}

S. Kukathasan and S. Pellegrino University of Cambridge, Cambridge, CB2 1PZ, UK

\section{4th AIAA/ASME/ASCE/AHS/ASC Structures, Structural Dynamics, and Materials Conference 7-10 April 2003 Norfolk, VA}

For permission to copy or republish, contact the American Institute of Aeronautics and Astronautics 1801 Alexander Bell Drive, Suite 500, Reston, VA 20191-4344 


\title{
NONLINEAR VIBRATION OF WRINKLED MEMBRANES
}

\author{
S. Kukathasan* ${ }^{*}$ and S. Pellegrino ${ }^{\dagger}$ \\ Department of Engineering, University of Cambridge \\ Trumpington Street, Cambridge, CB2 1PZ, U.K.
}

\begin{abstract}
This paper investigates the vibration behaviour of large, ultra-lightweight membrane structures. A highfidelity finite element simulation of a square membrane is set up, which uses thin-shell elements and a very careful simulation of the static wrinkling process. It produces natural modes and frequencies that are in excellent agreement with experimental measurements in air. This simulation is used as a benchmark for a lowerfidelity model, based on a linear-elastic material model of the membrane, and it is shown that the less accurate model is able to accurately predict the vibration of a moderately wrinkled membrane but not of a heavily wrinkled membrane. Nonlinear identification tests on a highly wrinkled membrane are also presented, showing that the existence of stiffness nonlinearities results in energy scattering and hence in an apparent increase of the damping of the structure when the amplitude of the excitation is increased.
\end{abstract}

\section{Introduction}

Most space membrane structures remain partially wrinkled, and hence are not perfectly smooth, in their operational configuration. Except for high precision applications, such as reflector antennas and space based radars which require unwrinkled, i.e. smooth surfaces, a wrinkled surface is acceptable for many applications. However, although wrinkled membranes are much easier to produce in practice than unwrinkled ones, accurately estimating the vibration behaviour of wrinkled membranes is much more challenging. A few modelling techniques have been recently proposed ${ }^{1}$, but so far without any experimental validation.

As a follow-on to our recent study of unwrinkled membrane vibration ${ }^{2}$ in this paper we present a finite element (FE) simulation technique that predicts with good accuracy the natural frequencies and mode shapes of wrinkled membrane structures, both in vacuum and in air. This technique is validated against experimental measurements in air.

Another important issue, recently reported, is that structures including wrinkled membranes exhibit vibration damping levels much larger than could possibly be due to material damping ${ }^{3,4}$. We have addressed this issue by means of an experimental investigation of the nonlinear vibration behaviour of heavily wrinkled membranes, which shows that their unually high vibration damping is actually caused by energy scattering into harmonics of the excitation frequency.

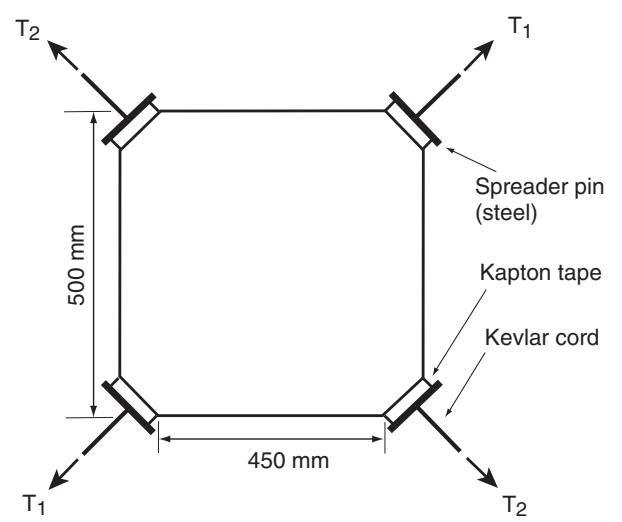

Figure 1: Schematic diagram of square membrane.

The layout of this paper is as follows. A brief review of the relevant literature is followed by a finite element study of the stress distribution and wrinkle pattern in the square membrane shown schematically in Figure 1, for different corner force ratios. The invacuum vibration of the membrane is shown to depend very significantly on the corner force ratio, which affects the wrinkle pattern. For $T_{1} / T_{2}=2$, there are only small wrinkles near the corners, and it is shown that a standard linear-elastic model of the membrane is adequate. For $T_{1} / T_{2}=4$ there are large diagonal wrinkles, and in this case it is essential to simulate the formation of wrinkles in the static analysis that

\footnotetext{
* Research Student, AIAA Student member.

${ }^{\dagger}$ Professor of Structural Engineering, Associate Fellow AIAA. pellegrino@eng.cam.ac.uk Copyright (c) 2003 by S. Pellegrino. Published by the American Institute of Aeronautics and Astronautics, Inc. with permission.
} 
precedes any vibration analysis. This work is then extended to the prediction of frequencies and mode shapes in-air, and the results are verified experimentally. Finally, for the case $T_{1} / T_{2}=4$ a sets of experiments on this highly wrinkled membrane are presented. A discussion concludes the paper.

\section{Background}

Wrinkled membranes cannot be analysed using standard linear-elastic membrane models, for a variety of reasons. From a purely static viewpoint, thin membranes are unable to carry compressive stresses due to their very low bending stiffness, and hence their stress distribution has to be purely tensile everywhere; also, the small bending stiffness of the membrane leads to the formation of finite-wavelength wrinkles in the surface. From a structural vibration viewpoint, a knowledge of the correct prestress distribution is obviously necessary to correctly model the out-of-plane stiffness distribution of the membrane.

Various analytical and numerical techniques have been developed to analyse wrinkled membrane structures; those most relevant to this study are briefly reviewed next.

\section{Static Analysis}

Highly wrinkled membranes, i.e. membranes subjected to in-plane edge loads or displacements well in excess of the magnitudes necessary to trigger out-of-plane buckling, were first studied using tension field theory ${ }^{5,6}$. In this approach, the bending stiffness of a membrane is neglected and hence no compressive stresses are allowed. Therefore, a uniaxial state of stress is assumed everywhere, defining a series of tension rays, whose orientation is such as to maximise the strain energy in the membrane. This approach has been used for the analysis of highly wrinkled membranes with simple shapes but, considering the complexity of the boundary shapes and load conditions that are of current interest, there is limited scope for using tension field theory.

An analytical formulation based on the concept of a variable Poisson's ratio for partially wrinkled membranes was proposed by Stein and Hedgepeth ${ }^{7}$. A partially wrinkled membrane is thus subdivided into wrinkled and taut regions. The membrane behaviour in the taut regions is estimated using linear elastic models, but in the wrinkled regions a variable Poisson's ratio is defined such that there are no compressive stresses.

A finite element implementation of the variable Poisson's ratio approach ${ }^{1,8}$ looks for any elements that satisfy the wrinkling criterion, to determine whether they stay taut or become wrinkled. For any element that becomes wrinkled, the element's stiffness is modi- fied according to the variable Poisson's ratio approach and the resulting model is re-analysed with the same loads until all compressive stresses are removed. This FE implementation is known as the Iterative Membrane Properties (IMP) procedure and has been recently implemented ${ }^{1}$ as a user subroutine in the commercial FE package ABAQUS ${ }^{9}$. This implementation has been used to analyse corner-loaded wrinkled membranes with different load and aspect ratios. The IMP approach is thus able to compute the extent and shape of the wrinkled regions and their in-plane stiffness, but not details of the wrinkles such as amplitudes and wavelength.

In all of the above approaches the membrane is treated as a purely two-dimensional structure. Rimrott and Cverko ${ }^{10}$ were the first to consider small outof-plane deformations, as well as the bending stiffness of the membrane, in a study of the wrinkles formed by a "blanket" hanging under gravity between two points. This approach was developed by Wong and Pellegrino ${ }^{11}$, and led to analytical expressions for the wavelength and amplitude of the diagonal wrinkles that form in long rectangular membranes subjected to shear. The analytical predictions were confirmed experimentally and also against simulations with $\mathrm{ABAQUS}^{12}$, where the membrane was modelled with shell elements, seeded with initial imperfections based on the bifurcation eigenmodes of the lightly prestressed membrane. To form an extensive set of wrinkles, a geometrically non-linear analysis was carried out, under displacement control, until the imposed edge displacement had been reached. This simulation technique will be extended in this paper to cornerloaded square membrane.

\section{Vibration Analysis}

The linear vibration behaviour of a wrinkled membrane can be easily predicted once a tangent stiffness matrix and a mass matrix have been obtained for the membrane, e.g. using an FE model of the membrane.

The natural frequencies and mode shapes of the Next Generation Space Telescope sunshield membrane were obtained using both a cable network model of the sunshield and a membrane modelled with the IMP approach $^{1}$. The cable network model approximates the membrane with a series of interconnected truss elements, representing the mass and load distribution in the membrane. The IMP procedure simulates the effects of the wrinkles, as explained in the previous section. Several test cases were analysed in Ref. 1 using both methods, but the results were not validated against any experiments.

Experiments on a large wrinkled membrane structure have shown that their vibration amplitude varies nonlinearly with the excitation ${ }^{3}$. In addition, modal 
damping coefficients obtained for a random excitation of $10 \mathrm{mg}$ rms were in the range $3-10 \%$, hence almost an order of magnitude higher than if material damping had been the only source of damping. On the other hand, unwrinkled membranes have been reported to behave linearly and to exhibit low damping ${ }^{2}$. An attempt to characterise the nonlinear behaviour of this wrinkled structure by means of nonlinear identification tests were inconclusive ${ }^{3}$, which provided the inspiration for the investigation of the nonlinear mechanisms behind the vibration behaviour of wrinkled membrane structures, described in the latter part of this paper.

\section{In-Vacuum FE Analysis}

Finite element analyses of the square membrane shown in Figure 1 were carried out. This is a square Kapton foil with side lengths of $0.5 \mathrm{~m}$ and a thickness of $25 \mu \mathrm{m}$. It is subject to pairs of equal and opposite corner forces $T_{1}, T_{2}$, where $T_{1} \geq T_{2}$. Two different values of this force ratio were considered, $T_{1} / T_{2}=2$ and $T_{1} / T_{2}=4$, to study the effects of different wrinkling patterns.

The corner forces are applied using $0.92 \mathrm{~mm}$ diameter Kevlar cords attached to the corners of the membrane through $1.5 \mathrm{~mm}$ diameter steel pins which spread the corner forces through $35 \mathrm{~mm}$ wide Kapton tape. An extra mass of $2.5 \mathrm{~g}$ is attached to a corner of the membrane, to simulate the effect of an accelerometer. All material properties of the model are defined in Table 1.

Table 1: Material properties of membrane.

\begin{tabular}{lccc}
\hline \hline Parameter & Membrane & Cord & Pin \\
\hline Material & Kapton & Kevlar & Steel \\
Density $\left(\mathrm{kg} / \mathrm{m}^{3}\right)$ & 1500 & 1450 & 7840 \\
Young's Modulus $(\mathrm{GPa})$ & 3.5 & 131 & 210 \\
Poisson's Ratio & 0.3 & 0.3 & 0.3 \\
\hline \hline
\end{tabular}

The wrinkled membrane was modelled using standard thin shell elements available in ABAQUS. Preliminary analyses led to the conclusion that secondorder elements are unsuitable for the wrinkling analysis, which is highly nonlinear. Therefore, the firstorder 3-node triangular (S3R) and 4-node quadrilateral (S4R5) thin shell elements were used in all analyses described in this paper. The cords were modelled with truss elements (T3D2) and the steel pins with beam elements (B31). The Kapton tape was also modelled using shell elements. All in-plane translations and out-of-plane rotations of the centre node of the membrane were constrained, to avoid rigid body motions due to small numerical asymmetries.

\section{Analysis Details}

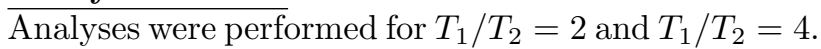

Under approximately equal corner forces the membrane is essentially taut everywhere, except for small wrinkled regions near the corners, but for $T_{1} / T_{2}>3$ a large diagonal wrinkle appears with small slack regions near the edges of the membrane. Two different static analyses were performed on the membrane for each corner load ratio, followed in each case by a linear frequency analysis.

\section{(i) Wrinkled Model}

The wrinkled model provides a close approximation to the actual membrane, both in terms of its shape and stress distribution. The static analysis, based on Ref. 12 was divided into three stages, as follows.

First, a uniform biaxial prestress of $0.5 \mathrm{~N} / \mathrm{mm}^{2}$ was applied to the membrane (to avoid numerical singularities in the following analysis) and then a buckling analysis was performed by applying corner forces $T_{1}=30 \mathrm{~N}$ and $T_{2}=7.5 \mathrm{~N}$. The amount of prestress and the magnitude of the corner forces are not important, as the aim of the buckling analysis is to provide a reasonable estimate of the geometric imperfections to be used as an input into the geometrically nonlinear post-buckling analysis, to trigger the formation of wrinkles. A linear combination of five eigenmodes similar to the expected wrinkling pattern was selected for use as an initial geometric imperfection.

Second, a geometrically non-linear post-buckling analysis was carried out under increasing corner forces, and with numerical stabilisation provided through the STABILIZE option in ABAQUS. This option automatically sets up viscous forces which are used in a pseudo-dynamic integration of the equations of motion of the structure. While the response of the membrane is stable, the viscous forces remain small because the nodal velocities are small, and hence equilibrium is not affected. When the model is unstable, the nodal velocities and hence also the viscous forces, become larger and thus stabilise the solution against numerical singularites. However, it has been found that if the viscous forces are too large, the final shape of the wrinkles is not accurate. Therefore, the damping factor has to be set to the smallest value for which convergence can be achieved. Its value was set at $2 \times 10^{-10}$ for most analyses.

Third, after performing the above two static analyses, a frequency analysis was carried out to estimate the natural frequencies and mode shapes.

\section{(ii) Unwrinkled Model}

The unwrinkled model is the simplest to analyse, as it provides a purely two-dimensional state of stress, although shell elements are used, because there is no mechanism to trigger any out-of-plane motion in the numerical solution. Therefore, in this case the static 
analysis does not predict any wrinkles. Although this approach cannot be expected to accurately predict the state of stress in highly wrinkled membranes, there are cases in which it is quite accurate. After applying the corner forces, a geometrically nonlinear static analysis was carried out in ABAQUS (to estimate the geometric stiffness due to the prestress) followed by a frequency analysis.

\section{Comparison of Wrinkled vs. Unwrinkled Models} The wrinkled and unwrinkled model results are compared for $T_{1} / T_{2}=2$ and $T_{1} / T_{2}=4$. First, the principal stress distributions are compared to identify the differences between the two analysis techniques for each load case. Also, the wrinkle patterns obtained from the wrinkled model are correlated with discrepancies in the stress distribution from the unwrinkled model. Finally, the natural vibration mode shapes predicted by both models are investigated.

(i) $\mathbf{T}_{1} / \mathbf{T}_{2}=\mathbf{2}$

The static and vibration analysis results from both models, for $T_{1}=30 \mathrm{~N}$ and $T_{2}=15 \mathrm{~N}$, are discussed in this section.

The major principal stress distribution from the wrinkled and unwrinkled models (not shown) are almost identical. Higher stresses tend to be concentrated near the corners, which indicates that the corner loads are transferred between corners, through most of the membrane surface.

The minor principal stress contours, plotted in Figure 2, show that both models predict compressive stresses at the corners with the larger load $\left(T_{1}=30\right.$ $\mathrm{N})$. However, note that the wrinkled model predicts a reduction in the compressive stresses along the diagonal subjected to the larger load.

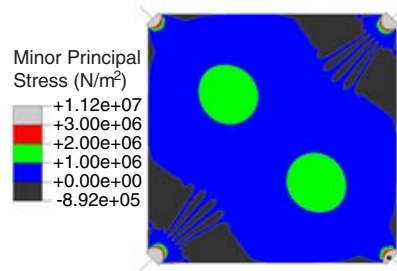

(a)

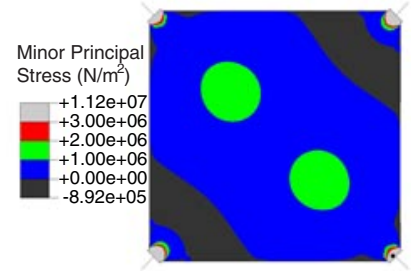

(b)
Figure 2: Mid-plane minor principal stresses for $T_{1} / T_{2}=2$ : (a) wrinkled and (b) unwrinkled models.

The wrinkle pattern from the wrinkled model is shown in Figure 3. This shows that smaller wrinkles form along the two opposite corners which are subject to the larger loads. The amplitude of the wrinkles is of the order of $10^{-2} \mathrm{~mm}$. Comparing the wrinkled shape in Figure 3 with the corresponding minor prin- cipal stress plot in Figure 2(a), it becomes clear that through the formation of wrinkles some of the midplane compressive stresses have been removed.

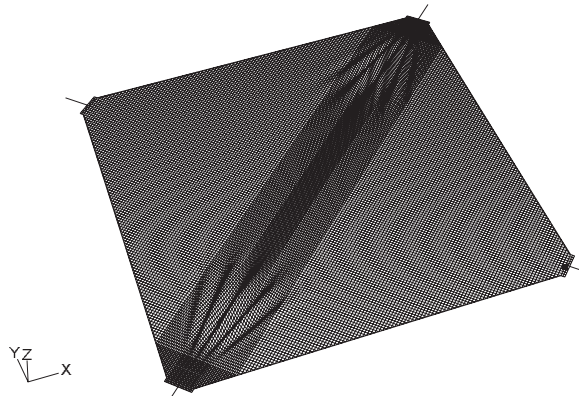

Figure 3: Wrinkle pattern for $T_{1} / T_{2}=2$.

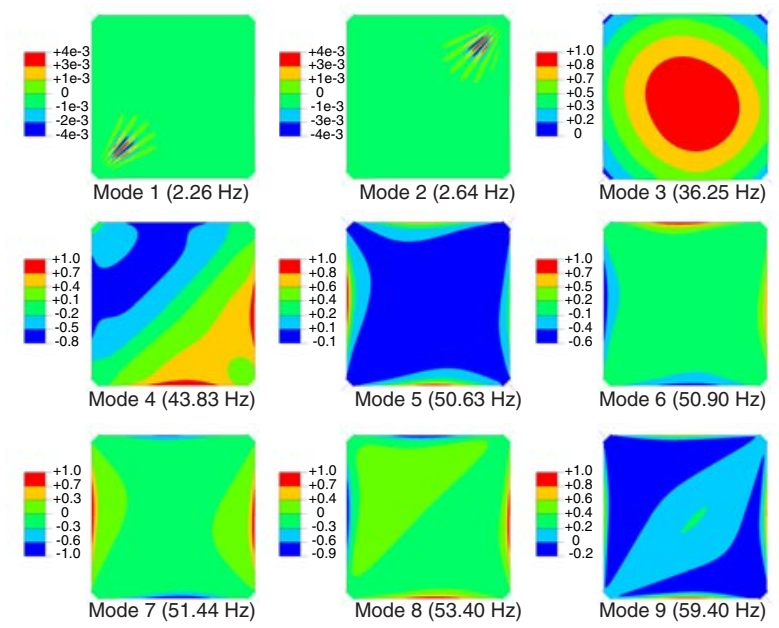

Figure 4: Wrinkled model mode shapes for $T_{1} / T_{2}=2$.

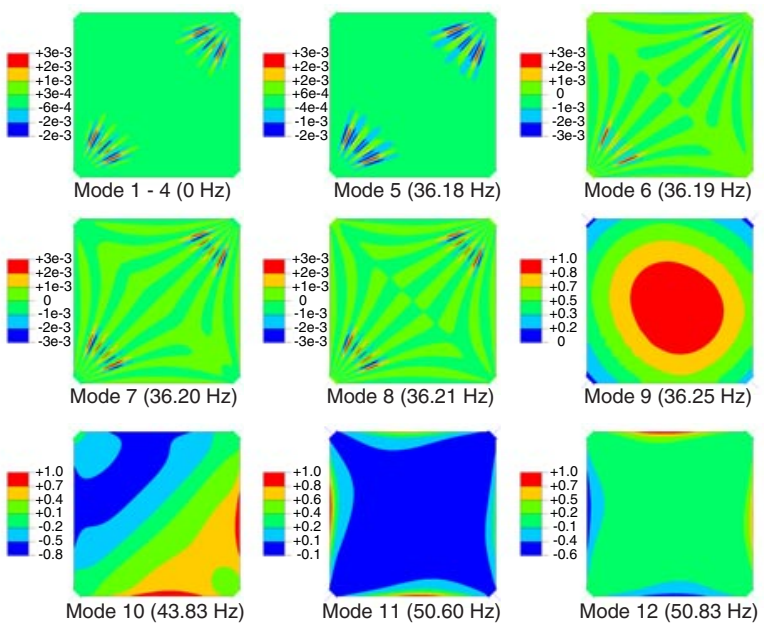

Figure 5: Unwrinkled model mode shapes for $T_{1} / T_{2}=$ 2 .

The first nine mode shapes obtained from the wrinkled model are plotted in Figure 4; note that modes 1-2 are local modes with very low frequency and negligible modal effective mass. Mode 3 is the dominant 
membrane mode with the largest modal effective mass in the out-of-plane direction.

The unwrinkled model predicts negative eigenvalues for the first four modes, one of which is plotted top-left in Figure 5 as a zero frequency mode. These modes, often called noise modes, appear as a result of negative geometric stiffness terms. Modes 5-8 are local membrane modes which have almost the same frequency; these four modes have very small modal effective mass in the out-of-plane direction. Mode 9 has the largest modal effective mass $(>80 \%)$ and appears to be the dominant global membrane mode.

The local, low frequency modes predicted by both models are due to small, residual compressive stresses which are left in the membrane during the postbuckling analysis.

Modes 3-6 predicted by the wrinkled model are practically identical, and have the same frequencies of modes 9-12 predicted by the unwrinkled model. Thus, it can be concluded that for $T_{1} / T_{2}=2$ the unwrinkled model accurately predicts the dominant membrane modes and hence can be used to predict the key modes using much smaller computer resources than the unwrinkled model.

(ii) $\mathbf{T}_{1} / \mathbf{T}_{2}=\mathbf{4}$

This section presents the results from the two models for $T_{1}=30 \mathrm{~N}$ and $T_{2}=7.5 \mathrm{~N}$.

The major principal stress distributions from the two models are significantly different. Figure 6(a) shows that the wrinkled model predicts corner stress distributions that join together along the diagonal with the larger load, whereas the stresses near the edges of the membrane are very small. This indicates that most of the corner tensions are transferred along the most heavily loaded diagonal. None of this is predicted by the unwrinkled model.

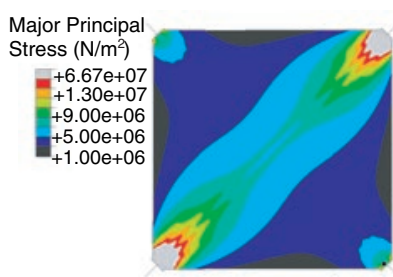

(a)

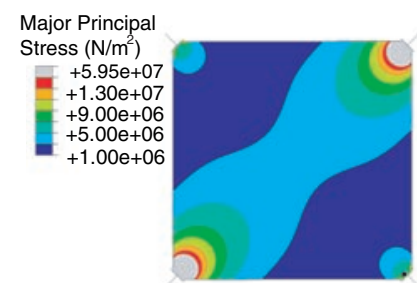

(b)
Figure 6: Major principal stress contours for $T_{1} / T_{2}=$ 4: (a) wrinkled and (b) unwrinkled models.

Figure 7 shows the minor principal stress distributions from the two models. The unwrinkled model predicts compressive stresses in large parts of the mem- brane, whereas the wrinkled model predicts compressive stresses only along the crests and troughs of the wrinkles.

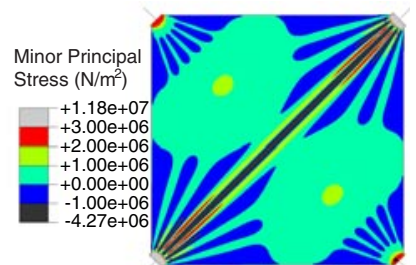

(a)

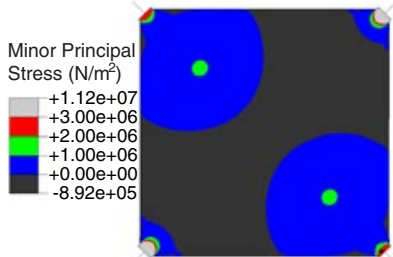

(b)
Figure 7: Minor principal stress contours for $T_{1} / T_{2}=$ 4: (a) wrinkled and (b) unwrinkled models.

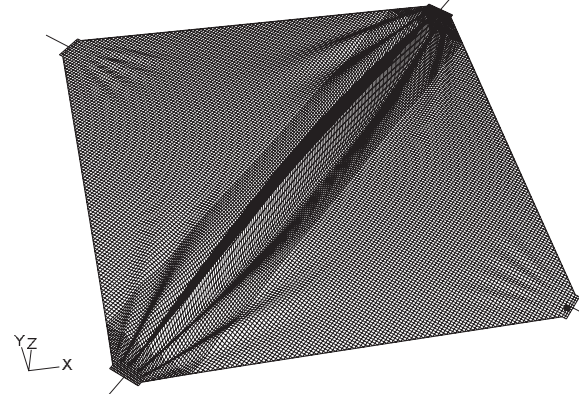

Figure 8: Wrinkle pattern for $T_{1} / T_{2}=4$.

It is clear that for $T_{1} / T_{2}=4$ the stress distribution predicted by the unwrinkled model is far from correct.

The wrinkled shape of the membrane predicted by the wrinkled model is plotted in Figure 8. It shows large amplitude diagonal wrinkles, together with small amplitude corner wrinkles along the two corners which carry the larger loads. Small corner wrinkles form near the corners which carry the smaller loads.

Figure 9 shows the first nine mode shapes predicted by the wrinkled model. Note that modes 1 and 2 are local modes, involving only the smaller corner wrinkles near the low-tensioned corners. Modes 3, 8 and 9 have large modal effective mass and hence are the dominant modes. Modes 4-6 are local modes only involving flapping of the membrane edges. Most of the other modes have similar features to those described above, having a small modal effective mass.

Figure 10 shows the first nine significant mode shapes predicted the unwrinkled model. Modes 1-16 are noise modes. Note that only modes 17 and 18 have large modal effective mass; most of the others involve almost randomly distributed motions of the membrane. This could be due to singularities arising from the presence of compressive stresses in the unwrinkled model. 


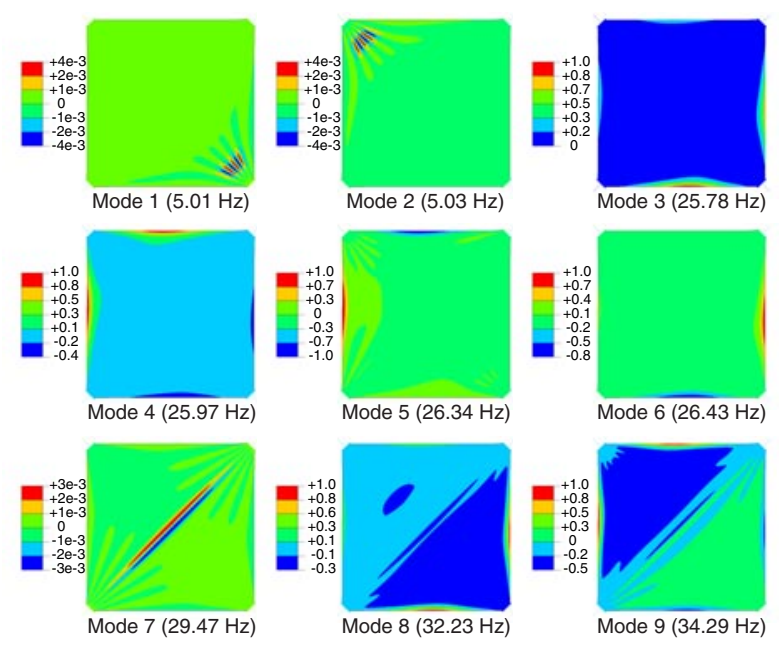

Figure 9: Wrinkled model mode shapes for $T_{1} / T_{2}=4$.
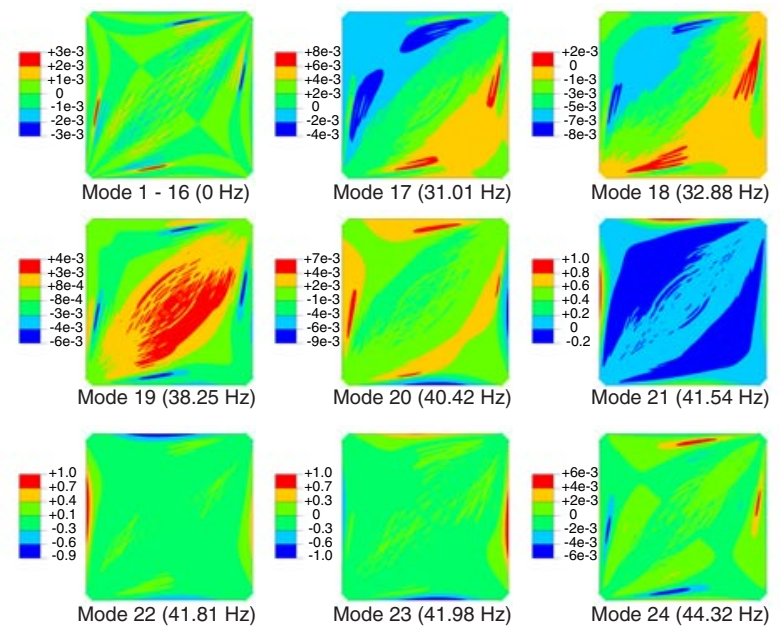

Figure 10: Unwrinkled model mode shapes for $T_{1} / T_{2}=4$

Most importantly, and unlike the case $T_{1} / T_{2}=2$, note that there is not even a single corresponding mode shape between the predictions from the two models, hence it can be concluded that for the case $T_{1} / T_{2}=4$ the unwrinkled model predictions are not useful.

\section{In-Air Finite Element Analysis}

The natural frequencies and mode shapes of a wrinkled membrane vibrating in air were estimated using the coupled acoustic-structural analysis described in Ref. 2. Analyses were carried out using the wrinkled model, for $T_{1} / T_{2}=2$ and $T_{1} / T_{2}=4$. In the modelling, a cubic air box with dimensions of $2.5 \times 2.5 \times 2$ $\mathrm{m}$ was defined with the membrane in the middle. The dimensions of the air box were determined from the in-vacuum fundamental frequency of the membrane, as discussed in Ref. 2.

First, a nonlinear static analysis was performed to obtain the wrinkled shape, without coupling with acoustic elements. Then, a linear dynamic analysis was performed after coupling the membrane with the acoustic elements. This analysis was performed by applying a unit load at the corner of the membrane where an accelerometer had been simulated, and by varying the frequency in the range $0-50 \mathrm{~Hz}$. The responses of all membrane nodes to this input were recorded and the natural frequencies of the membrane were identified by examining the frequency response at a selected point of the membrane. The first six identified mode shapes are plotted in Figures 11 and 12.

\section{Comparison of In-Air and In-Vacuum Modes}

The in-air modes for $T_{1} / T_{2}=2$, Figure 11 , are compared with the in-vacuum modes obtained from the wrinkled model, Figure 4. The first two modes in Figure 4 and also the local modes which involve flapping of the membrane edges, predicted by the in-vacuum model, do not appear in air. This shows that local modes with very low modal effective mass are suppressed by air mass effects.

On the other hand, modes 3-5 from the in-vacuum model, which are the dominant modes, agree fairly well with the first three mode shapes from the in-air model. Of course, the in-air frequencies of these three modes are much lower than the corresponding in-vacuum frequencies, due to the added air mass.
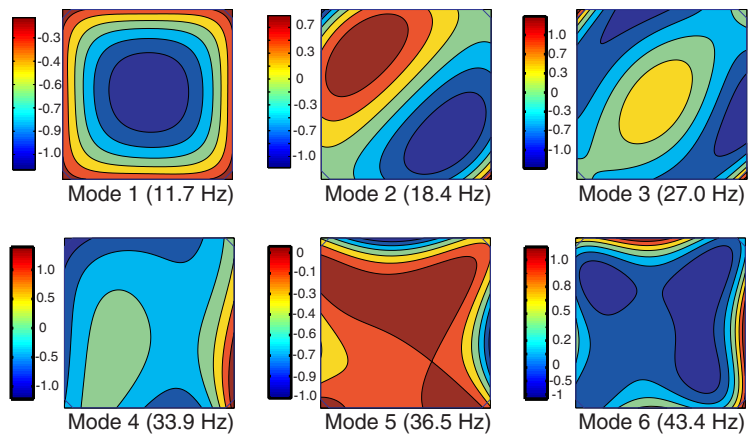

Figure 11: FE in-air mode shapes for $T_{1} / T_{2}=2$.
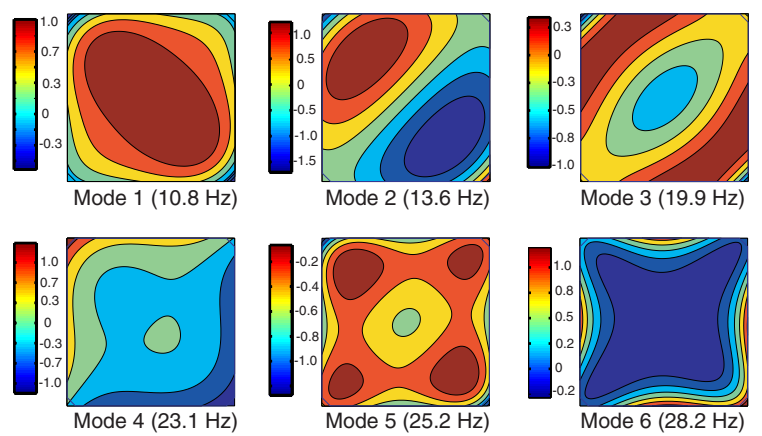

Figure 12: FE in-air mode shapes for $T_{1} / T_{2}=4$.

For $T_{1} / T_{2}=4$, the mode shapes in-air and invacuum, plotted in Figures 12 and 9 respectively, do 
not show any correlation. It was noted in the previous section that most in-vacuum modes are local and involve only very small effective mass. On the other hand, the in-air modes plotted in Figure 12 appear to be global membrane modes. This suggests that the vibration of membranes with large amplitude diagonal wrinkles is sensitive to the surrounding medium.

When the in-air modes for the two load ratios are compared, it can be seen that the first three mode shapes are fairly similar. This indicates that in-air modes may not be sensitive to even large changes of load path.

\section{Experiments}

An experimental investigation of the linear and nonlinear vibration behaviour of a corner-loaded square wrinkled membrane in air is described in this section. Two types of vibration tests were carried out: (i) a linear identification test using slow sine sweep and constant sine excitation, and (ii) a nonlinear identification test using slow sine sweep excitation. A Polytec PSV300 scanning laser vibrometer was used to measure the responses in both tests.

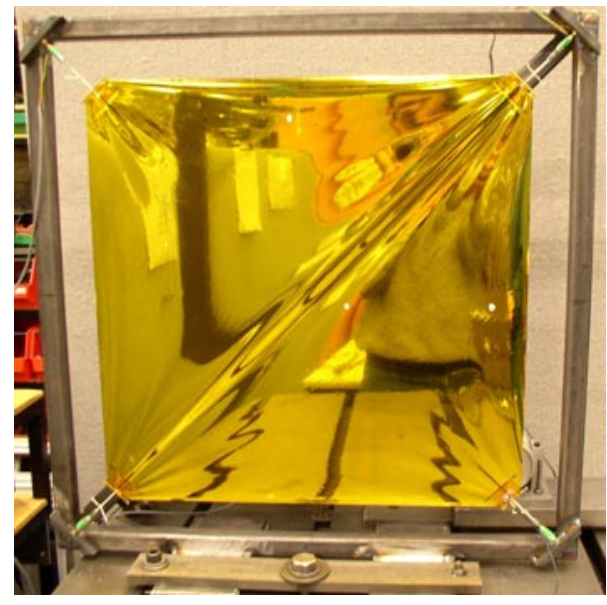

Figure 13: Photograph of experimental model with diagonal wrinkle.

The test model is shown in Figure 13 and its geometry was defined in the Section In-Vacuum FE Analysis. Note that the Kevlar cords connecting the membrane to the frame go through a strain-gauged turnbuckle which is used to tension the membrane.

The membrane was excited using an LDS V201 electromagnetic shaker, connected to the lower-right corner of the membrane through a stinger. The stinger was glued to the membrane and the acceleration input to the membrane was measured using a PCB M353 accelerometer (calibration $10.3 \mathrm{mV} / \mathrm{g}$ ) mounted in-line with the stinger, in front of the membrane. The accelerometer signal was amplified using a PCB 480E09 conditioning amplifier. A TG1241 waveform signal generator was used to provide the input signals to the shaker through a CA5220 power amplifier.

\section{Linear Identification}

The natural frequencies and mode shapes of the membrane with load ratios of 2 and 4 were identified in the range of $0-50 \mathrm{~Hz}$. The amplitude of the excitation was selected to be small enough to minimise any nonlinear effects. First, approximate natural frequencies were obtained using a broad-band sine sweep. Then, more accurate frequencies and damping values were obtained by performing a fine-scale sine sweep around the approximate natural frequencies. Finally, the mode shapes of the membrane were measured by scanning the surface with the laser vibrometer while exciting the model at a constant frequency.

\section{(i) Natural Frequencies}

Broad-band sine sweep excitation, with an amplitude of approximately $2.4 \mathrm{~m} / \mathrm{s}^{2}$, was applied by the shaker to obtain a frequency-response function from which the approximate resonant frequencies of the membrane could be estimated. The response was measured at a point near the centre of the membrane, slightly away from the diagonal wrinkle. A linear sine sweep ranging from 0 to $50 \mathrm{~Hz}$ over 100 seconds was applied using the waveform generator and the response was sampled at $100 \mathrm{~Hz}$. Each test was repeated five times and the results were averaged to reduce the signal-noise ratio. The frequency-response functions were derived using a MATLAB program.

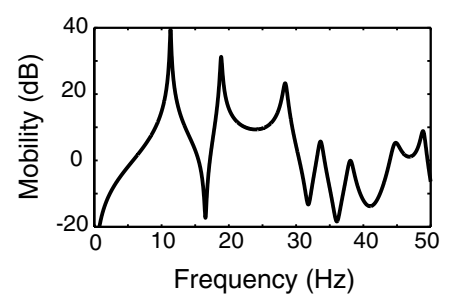

(a)

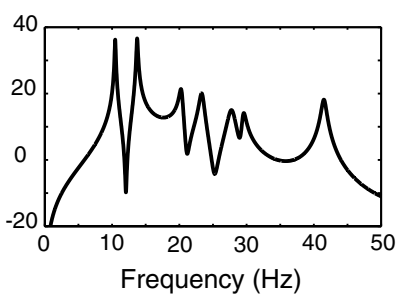

(b)
Figure 14: Frequency-response plots for (a) $T_{1} / T_{2}=2$ and (b) $T_{1} / T_{2}=4$.

Then, narrow-band frequency sweeps were used to obtain accurate resonant frequencies and damping values. Frequency bandwidths of $2 \mathrm{~Hz}$ around each approximate frequency were swept at a linear rate over 50 seconds. The response of the membrane was sampled at $100 \mathrm{~Hz}$. The natural frequencies and damping factors obtained by fitting circles to the Nyquist plots are tabulated in Table 2. Frequency-response plots obtained from the broad-band sine sweep test, 
for $T_{1} / T_{2}=2$ and $T_{1} / T_{2}=4$, are reconstructed using the accurate frequencies and damping values obtained from the narrow-band sweep, and are plotted in Figure 14 .

Table 2 shows that the modal damping ratios, $\zeta$, of the wrinkled membrane for $T_{1} / T_{2}=2$ range from $0.61 \%$ to $1.71 \%$ while for $T_{1} / T_{2}=4$ they range from $0.77 \%$ to $2.05 \%$. The average damping ratio for the higher load ratio is slightly higher.

Table 2: Natural frequencies and damping ratios.

\begin{tabular}{ccccc}
\hline \hline \multirow{2}{*}{ Mode } & \multicolumn{2}{c}{$T_{1} / T_{2}=2$} & \multicolumn{2}{c}{$T_{1} / T_{2}=4$} \\
\cline { 2 - 5 } & $f(\mathrm{~Hz})$ & $\zeta(\%)$ & $f(\mathrm{~Hz})$ & $\zeta(\%)$ \\
\hline 1 & 11.3 & 0.61 & 10.4 & 0.77 \\
2 & 18.6 & 0.68 & 13.5 & 0.70 \\
3 & 28.0 & 1.04 & 20.2 & 1.52 \\
4 & 33.2 & 1.20 & 23.1 & 1.37 \\
5 & 37.4 & 0.95 & 27.7 & 2.05 \\
6 & 44.4 & 1.71 & 29.1 & 1.42 \\
\hline \hline
\end{tabular}

\section{(ii) Mode Shapes}

The mode shapes of the membrane were obtained by measuring the responses at 300 target points, regularly spaced throughout the surface, for 2 seconds at each point.

Contour plots of the first six identified modes - or, more correctly, the experimental operating deflection shapes (ODS) - of the membrane are plotted in Figures 15 and 16 .

Table 3: In-air FE and experimental frequencies.

\begin{tabular}{ccccccc}
\hline \hline \multirow{2}{*}{ Mode } & \multicolumn{3}{c}{$T_{1} / T_{2}=2$} & \multicolumn{3}{c}{$T_{1} / T_{2}=4$} \\
\cline { 2 - 7 } & $f(\mathrm{~Hz})$ & Error & \multicolumn{2}{c}{$f(\mathrm{~Hz})$} & Error \\
\cline { 2 - 7 } & $\mathrm{FE}$ & $\operatorname{Exp}$ & $(\%)$ & $\mathrm{FE}$ & $\operatorname{Exp}$ & $(\%)$ \\
\hline 1 & 11.7 & 11.3 & 3.4 & 10.8 & 10.4 & 3.7 \\
2 & 18.4 & 18.6 & -1.1 & 13.6 & 13.5 & 0.7 \\
3 & 27.0 & 28.0 & -3.7 & 19.9 & 20.2 & -1.5 \\
\hline \hline
\end{tabular}

Compare the in-air experimental and FE mode shapes for $T_{1} / T_{2}=2$, Figures 15 and 11 . The first three mode shapes are almost identical with only minor differences in the corresponding frequencies. Similarly, the first three mode shapes for $T_{1} / T_{2}=4$, plotted in Figures 16 and 12, also show good correlation with minor differences in frequencies.

Table 3 shows that the frequencies obtained from the in-air FE simulation are within $4 \%$ of the experimentally measured frequencies.
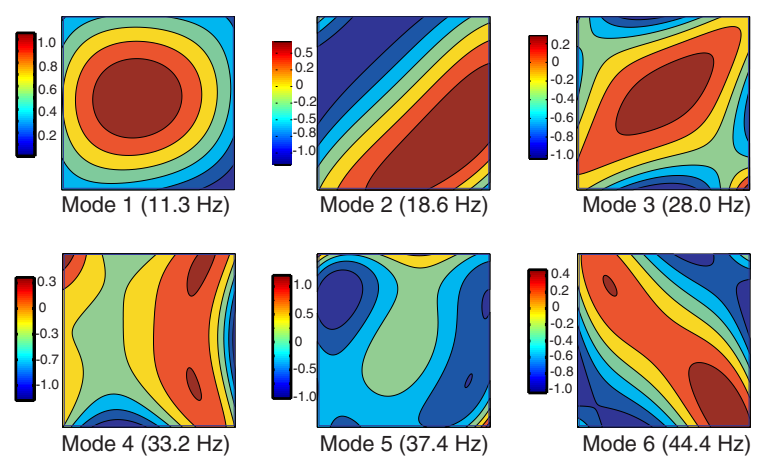

Figure 15: Experimental in-air mode shapes for $T_{1} / T_{2}=2$.
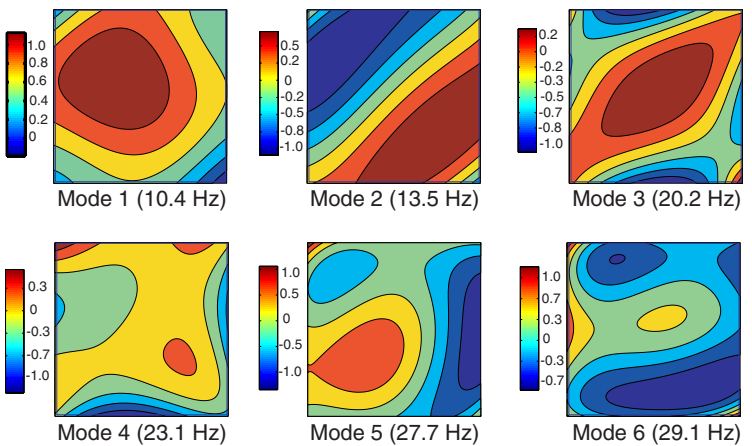

Figure 16: Experimental in-air mode shapes for $T_{1} / T_{2}=4$.

\section{Nonlinear Identification}

Nonlinear vibration behaviour of the membrane occurs under corner loads $T_{1}=30$ and $T_{2}=7.5 \mathrm{~N}$ (load ratio 4). First, the overall behaviour of the model was studied in the frequency range $0-30 \mathrm{~Hz}$, which includes the first six identified modes of the membrane. A slow sine sweep input was used to excite the membrane while measuring the response of a particular target point. The results from this preliminary test show the presence of harmonics in each mode of vibration. Therefore, a detailed study of the first three modes was carried out, to understand the presence of harmonics of the primary resonances. In this study, frequencyresponse plots of the primary resonances and power spectrum of the output signals were plotted for three levels of excitation, to better characterise the underlying nonlinear mechanisms.

\section{(i) Overall Behaviour}

The nonlinear behaviour of the wrinkled membrane was investigated by applying three different amplitudes of broad-band sine sweep excitation. These amplitudes will be referred to as low, medium and high, and are $4.74,9.48$ and $18.96 \mathrm{~m} / \mathrm{s}^{2}$, respectively. 


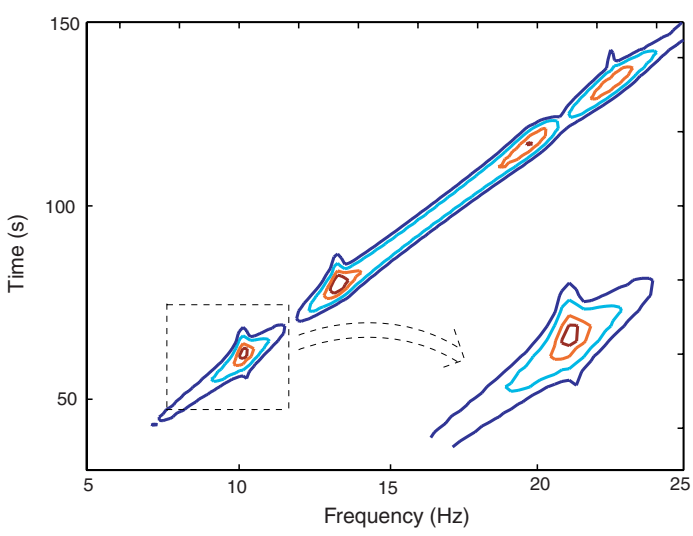

Figure 17: Sonogram for membrane with $T_{1}=30$ and $T_{2}=7.5 \mathrm{~N}$.

The frequency range $0-30 \mathrm{~Hz}$ was swept linearly over 180 seconds while measuring the response at a target point near the diagonal wrinkle. The results are presented in Figure 18 as a "sonogram"13, i.e. as a plot of power-spectral amplitude of the experimentally measured response against time and frequency. A sonogram is, in effect, a collection of Fourier transforms for successive time windows, which maps out the response of the membrane at continuously varying excitation frequencies.

The sonogram for response of the membrane with low amplitude of excitation is plotted in Figure 17 for the frequency range $5-25 \mathrm{~Hz}$. On the vertical axis of the contour plot one can plot the time corresponding to each particular time window, as shown in Figure 17, or the excitation frequency at each particular time, as shown in Figure 18. The resonant frequencies of the membrane are identified by the peaks in the sonogram. For example, in Figure 17 the first peak response is at around $10.5 \mathrm{~Hz}$.

This type of plot clearly shows any harmonics of the resonant frequencies, and it is interesting to compare what happens when the excitation amplitude is increased from low, to medium and then to high; the corresponding sonograms are shown in Figure 18. This time the sonogram is plotted in the frequency range 0-90 Hz, to identify the harmonics. In the frequency range $0-30 \mathrm{~Hz}$, which corresponds to the excitation frequencies, there are six peaks as marked in Figure 18(a). These peaks correspond to the primary resonances of the membrane.

Note that above $10 \mathrm{~Hz}$ the response at the driving frequency forms almost continuous contours. The contours of the harmonic response at twice the driving frequency change from clearly discontinuous at low excitation, to almost continuous as the excitation is increased. Clearly, the amount of energy scattering, responsible for the harmonic response, increases. This indicates that the response of the membrane at the excitation frequency would appear to be much more highly damped.
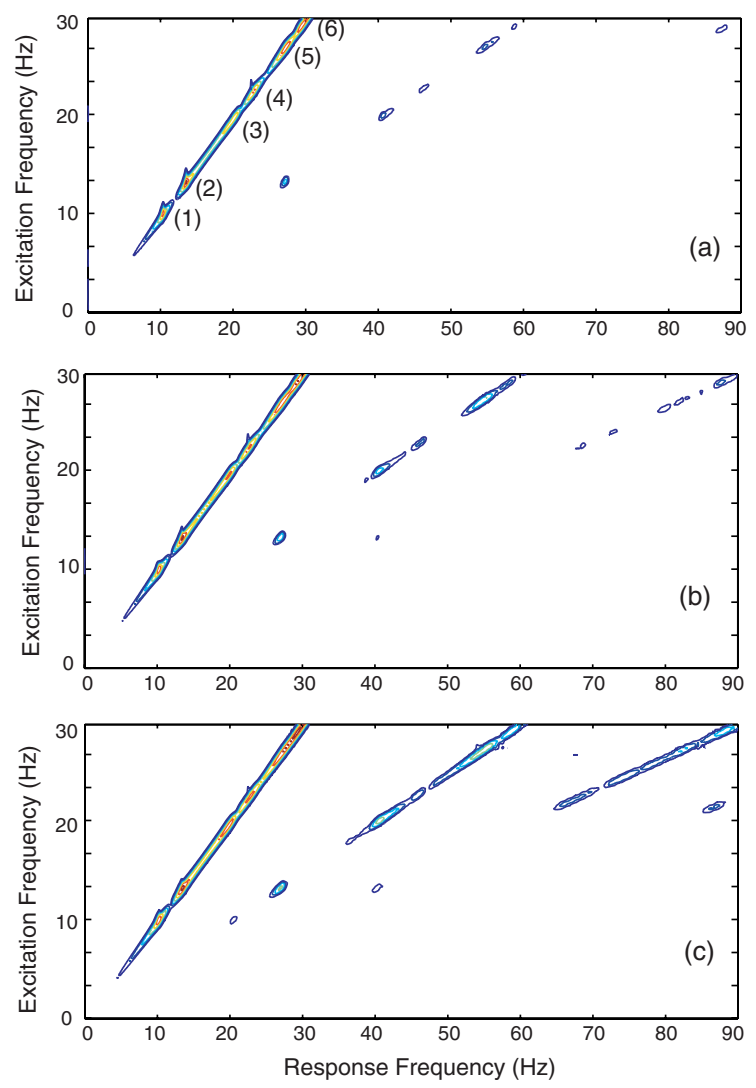

Figure 18: Sonograms of membrane with corner loads $T_{1}=30$ and $T_{2}=7.5 \mathrm{~N}$ for (a) low, (b) medium, and (c) high excitation levels.

\section{(ii) Detailed Behaviour}

Detailed tests around the first three natural frequencies were carried out to explore the nonlinear behaviour observed in the previous overall study. In the test, the response of the membrane was measured at a target point by applying fine-scale sine sweep excitation. An input band of $2 \mathrm{~Hz}$ around the natural frequencies of the first three modes identified in the linear test, was swept over 60 seconds. The resonant frequencies and damping ratios of the first three modes, for three different excitation amplitudes are listed in Table 4.

Table 4 shows that the damping increases in all three modes as the amplitude of excitation increases. On the other hand, the frequency variation differs for each mode. The first mode has almost the same frequency of vibration at all excitation levels, whereas the second and third mode frequencies increase slightly with the increase in the amplitude level of excitation. 
Table 4: Experimental natural frequencies and damping ratios for $T_{1} / T_{2}=4$.

\begin{tabular}{clcc}
\hline \hline \multirow{2}{*}{ Mode } & Input Force & $f(\mathrm{~Hz})$ & $\zeta(\%)$ \\
\hline \multirow{2}{*}{1} & Low & 10.39 & 1.12 \\
& Medium & 10.38 & 1.54 \\
& High & 10.38 & 2.10 \\
\hline \multirow{2}{*}{2} & Low & 13.48 & 1.02 \\
& Medium & 13.50 & 1.51 \\
& High & 13.59 & 2.13 \\
\hline \multirow{2}{*}{3} & Low & 20.30 & 1.61 \\
& Medium & 20.31 & 2.11 \\
& High & 20.44 & 2.75 \\
\hline \hline
\end{tabular}

Frequency-response plots of the second mode for three different excitation levels are plotted in Figure 19. When the amplitude of excitation is increased from low to medium and then to high, the resonance frequency increases from $13.48 \mathrm{~Hz}$ to $13.50 \mathrm{~Hz}$ and then to $13.59 \mathrm{~Hz}$, respectively. In addition, the peak amplitude reduces from around $20 \mathrm{~dB}$ to $18 \mathrm{~dB}$ and then to $14 \mathrm{~dB}$, and the damping increases from $1.02 \%$ to $1.51 \%$ and then to $2.13 \%$. This shows that the response of the membrane is characterised by a hardening nonlinearity ${ }^{14,15}$.

The output responses at the resonance frequency with each amplitude of excitation were investigated to identify the nonlinearity more clearly. Figure 20 shows the output spectra of the second resonant frequency, at the three excitation levels. When the amplitude of excitation is increased, both the amplitude and the order of the harmonics present in the response increase. The presence of the second and third harmonics of the excitation frequency shows the existence of both quadratic and cubic stiffness nonlinearities in the response of the wrinkled membrane, when it is excited in the second mode.

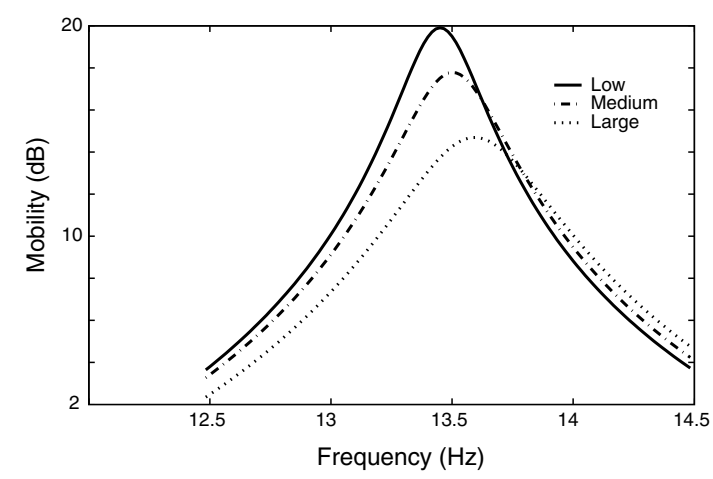

Figure 19: Frequency-response plot for mode 2.

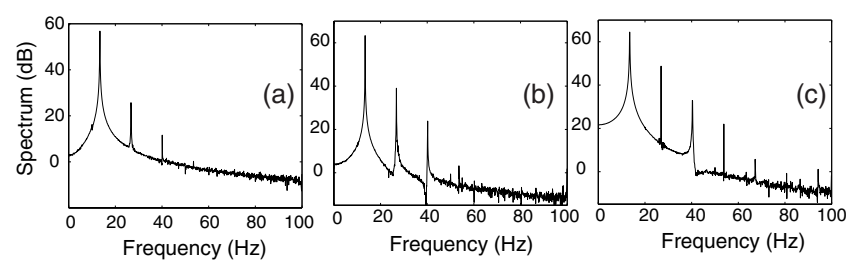

Figure 20: Output spectrum for mode 2 with (a) low, (b) medium and (c) large excitations.

\section{Conclusion}

The in-air FE modes and natural frequencies obtained from a high-fidelity simulation of a wrinkled membrane, which uses thin-shell elements and a very careful simulation of the static wrinkling process, are in excellent agreement with the experiments that have been carried out.

The results from this type of model have been used as a benchmark for a lower-fidelity model, and have shown that a linear-elastic model of the membrane (unwrinkled model) is able to accurately predict the vibration of a moderately wrinkled membrane (load ratio of 2), but not of a heavily wrinkled membrane (load ratio of 4), both in vacuum and in air.

The key problem of the unwrinkled model considered in this paper is that it fails to properly capture the stress distribution in highly wrinkled membranes. However, it seems likely that a more refined two-dimensional model of the membrane, e.g. based on the IMP formulation, should be adequate.

The nonlinear identification tests on a highly wrinkled membrane reported in this paper have shown the existence of quadratic and cubic nonlinearities in the stiffness of the membrane. These result in scattering of the energy input into higher harmonics and hence in an apparent increase of the damping of the structure. In our tests, the average damping ratios for the first three global membrane modes increased from $1.2 \%$ to $2.3 \%$ when the amplitude of the excitation was increased by a factor of 4 .

\section{Acknowledgments}

We thank Professors K.C. Park and J. Woodhouse for helpful comments. Partial support from NASA Langley Research Center, research grant NAG-1-02009, Integrated membranous-microelement space structures technology (technical monitor Dr. K. Belvin) is gratefully acknowledged.

\section{References}

1 Adler, A. L. Finite Element Approaches for Static and Dynamic Analysis of Partially Wrinkled Membrane Structures, Ph.D. Dissertation, University of 
Colorado at Boulder, 2000.

2 Kukathasan, S., and Pellegrino, S., "Vibration of Prestressed Membrane Structures in Air", Proc. $43 \mathrm{rd}$ AIAA/ASME/ASCE/AHS/ASC Structures, Structural Dynamics, and Materials Conference and Exhibit, Denver, CO, 2002, AIAA-2002-1368.

3 Lienard, S., Johnston, J.D., Ross B.P., and Smith, J., "Dynamic testing of a subscale sunshield for the Next Generation Space Telescope", Proc. 42nd AIAA/ASME/ASCE/AHS/ASC Structures, Structural Dynamics, and Materials Conference and Exhibit, Seattle, WA, 2001, AIAA-2001-1268.

4 Pappa, R.S., Lassiter, J. O., and Ross, B.P., "Structural dynamic experimental activities in ultralightweight and inflatable space structures", Proc. 42nd AIAA/ASME/ASCE/AHS/ASC Structures, Structural Dynamics, and Materials Conference and Exhibit, Seattle, WA, 2001, AIAA-2001-1263.

5 Mansfield, E.H., "Tension field theory, a new approach which shows its duality with inextensional theory", XII Int. Cong. of Applied Mechanics, pp. 305-320, 1968.

6 Mansfield, E.H., "Load Transfer via a Wrinkled Membrane", Proceedings of Royal Society of London, A316, pp. 269-289, 1970.

7 Stein, M. and Hedgepeth, J.M., "Analysis of partly wrinkled membranes", NASA TN D-813

8 Miller, R.K., Hedgepeth, J.M., Weingarten, V.I.,
Das, P. and Kahyai, S., "Finite element analysis of partly wrinkled membranes", Computers and Structures, Vol. 20, pp. 631-639, 1985.

9 ABAQUS, Inc., ABAQUS Standard User's Manuals, Version 6.2, Pawtucket, RI, USA, 2001.

${ }^{10}$ Rimrott, F.P.J. and Cvercko, M., "Wrinkling in thin plates due to in-plane body forces", IUTAM Symposium on Inelastic behaviour of Plates and Shells, Rio De Janeiro, 1985.

${ }^{11}$ Wong, Y.W. and Pellegrino, S., "Amplitude of wrinkles in thin membranes", New Approaches to Structural Mechanics, Shells and Biological Structures, pp. 257-270, Kluwer Academic Publishers, 2002.

12 Wong, Y.W., and Pellegrino, S., "Computation of Wrinkle Amplitudes in Thin Membranes", Proc. 43rd AIAA/ASME/ASCE/AHS/ASC Structures, Structural Dynamics, and Materials Conference and Exhibit, Denver, CO, 2002, AIAA-2002-1368.

13 Hodges, C.H., Power, J., Woodhouse, J., "The Use of Sonogram in Structural Acoustics and an Application to the Vibrations of Cylindrical Shells", Journal of Sound and Vibration, Vol. 101, pp. 203-218, 1985.

14 Nayfeh, A.H., and Moon, D.T., Nonlinear Oscillations, Wiley Inc., 1995.

15 Worden, K., and Tomlinson, G.R., Nonlinearity in Structural Dynamics, Institute of Physics Inc., 2001. 